\title{
Construction of Accurate Crack Identification on Concrete Structure using Hybrid Deep Learning Approach
}

Edriss Eisa Babikir Adam,

Assistant Professor / EEE,

Mainefhi College of Engineering and Technology,

Mainefhi, Eritrea.

Email: bonzoga20@gmail.com

\section{A. Sathesh, \\ Department of EEE, \\ Eritrea Institute of Technology, \\ Eritrea. \\ Email: sathesh4you@gmail.com}

Abstract- In general, several conservative techniques are available for detecting cracks in concrete bridges but they have significant limitations, including low accuracy and efficiency. Due to the expansion of the neural network method, the performance of digital image processing based crack identification has recently diminished. Many single classifier approaches are used to detect the cracks with high accuracy. The classifiers are not concentrating on random fluctuation in the training dataset and also it reflects in the final output as an over-fitting phenomenon. Though this model contains many parameters to justify the training data, it fails in the residual variation. These residual variations are frequent in UAV recorded photos as well as many camera images. To reduce this challenge, a noise reduction technique is utilized along with an SVM classifier to reduce classification error. The proposed technique is more resourceful by performing classification via SVM approach, and further the feature extraction and network training has been implemented by using the $\mathrm{CNN}$ method. The captured digital images are processed by incorporating the bending test through reinforced concrete beams. Moreover, the proposed method is determining the widths of the crack by employing binary conversion in the captured images. The proposed model outperforms conservative techniques, single type classifiers, and image segmentation type process methods in terms of accuracy. The obtained 
Journal of Innovative Image Processing (JIIP) (2021)

Vol.03/ No. 02

Pages: 85-99

https://www.irojournals.com/iroiip/

DOI: https://doi.org/10.36548/jiip.2021.2.002

results have proved that, the proposed hybrid method is more accurate and suitable for crack detection in concrete bridges especially in the unmanned environment.

Keywords: Crack identification techniques, Machine language

\section{INTRODUCTION}

In concrete structures, the crack is one of the most dangerous defects when compared to all other defects, which can destroy the building. This crack will affect the appearance of the building structure and it will be influenced by corrosion of the internal materials [1]. Since there is no visibility on the exterior side of the building's wall when the fracture is small or originates from the interior of the material, it is difficult to identify them beforehand. [2].

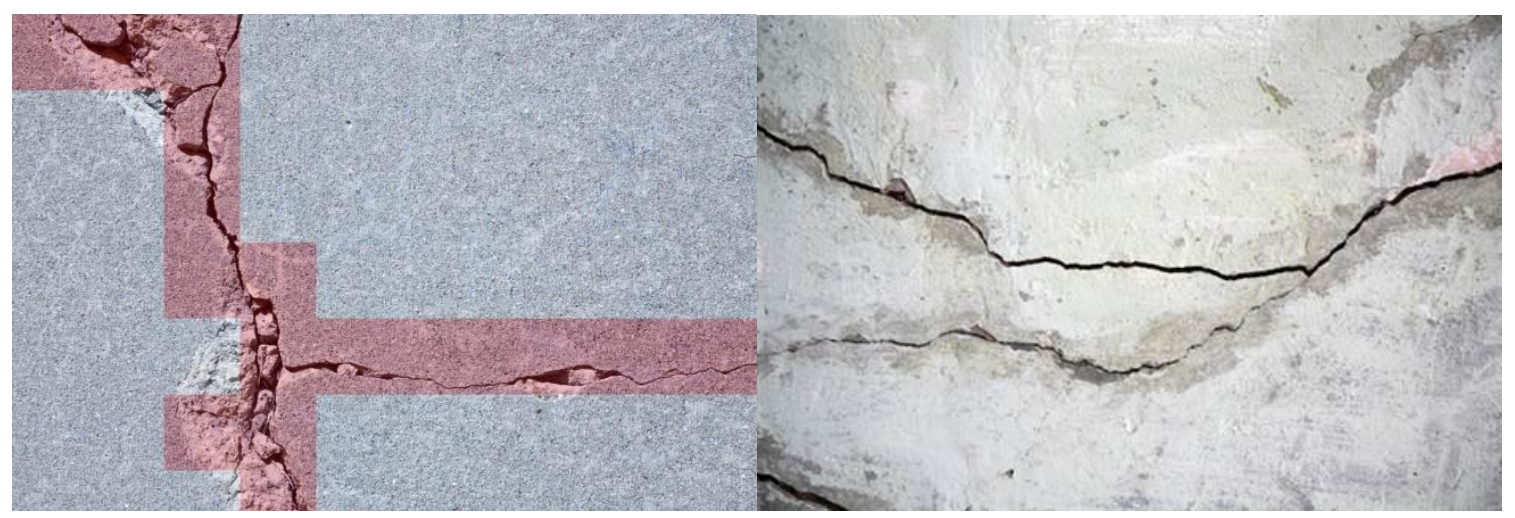

Figure 1 samples of crack images on the concrete structure

For safety analysis and assessment of concrete bridges, the earlier crack detection with good accuracy will be suitable and it is extremely important. Fluorescent microscopy and scanning electron microscopy are used to detect a crack in concrete bridges even on buildings in ancient times [3]. After a long time, the digital image processing techniques are mostly used to detect the crack on the building and concrete bridges. Image threshold techniques are used in digital image processing for crack detection. The cracking issue is present due to many reasons for concrete structures [4]. Categorizing the cracking severity is a kind of future prediction of concretes on bridges. 
Journal of Innovative Image Processing (JIIP) (2021)

Vol.03/ No. 02

Pages: 85-99

https://www.irojournals.com/iroiip/

DOI: https://doi.org/10.36548/jiip.2021.2.002

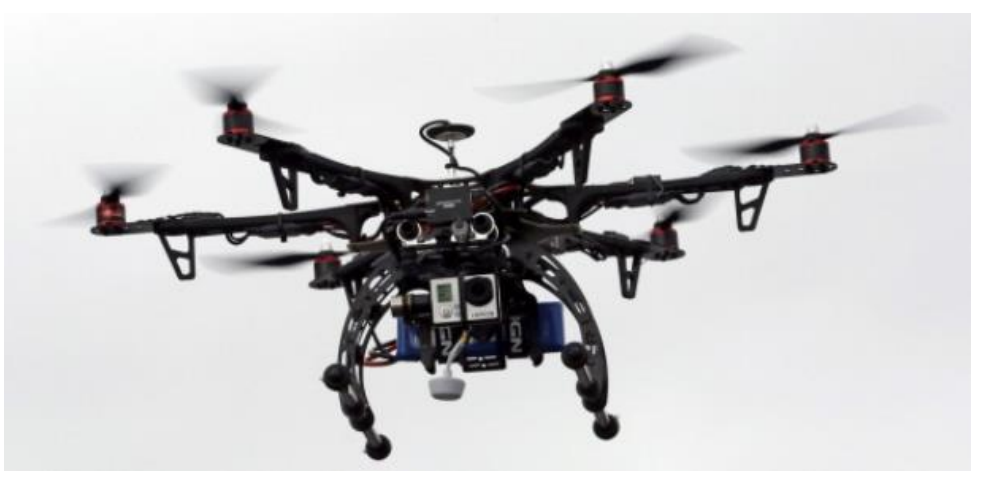

Figure 2 Drone setup for crack identification in unmanned area

The predicting crack in the concrete bridges is an essential process on civil works in many countries for safety measures. Due to reinforcement corrosion and sudden changing climatic condition, cracks can perform on the concrete bridges. The monitoring of cracks for the concrete structure will be an effective technique to prepare the appropriate safety measures for further examination [5]. Recently, many countries are inspecting crack detection with a drone in an effective manner. Because the human visual inspection is the ancient method and it is a trustworthy method of course. But, this type of inspection is very expensive in terms of time and labor charge in many developed countries. Some high-temperature nations, such as the United Arab Emirates (UAE), are reducing manual human eye inspection and shifting to a variety of drone-based crack detection technologies. This unmanned detection technique will reduce labour costs and time to perform a more effective work. [6]. Thus, these inspection methods are providing more efficiency and accuracy for performing crack detection by implementing unmanned aerial vehicles (UAV). The developed drone device is equipped with various goodquality cameras, sensors, and accurate GPS device. This experimental setup is more popular in Arab countries nowadays due to its ability to completely replace human labor [7]. This drone setup is used to detect the crack on concrete bridges and it is shown in the figure 2 . The process is much formulated in this device, where the captured images are processed by utilizing many effective images processing algorithms to detect a crack [8]. This process is used to detect crack and the crack identified scenario will be reported to the station. The colour image processing is suitable for obtaining appropriate images from the UAVs camera with sufficient information. Each picture from the camera provides various dimensions of the cracks [9]. 
Journal of Innovative Image Processing (JIIP) (2021)

Vol.03/ No. 02

Pages: 85-99

https://www.irojournals.com/iroiip/

DOI: https://doi.org/10.36548/jiip.2021.2.002

\section{ORGANIZATION OF THE RESEARCH}

This is the structure of the research article; the current research on crack detection on buildings is presented in Section 3. The proposed method for identifying building cracks without error is described in section4. Section 5 offers a different comparison of research with the method we are proposing. Section 6 will discuss the conclusion and the future task.

\section{PRELIMINARIES}

Alam et al combined correlation and acoustic emission for digital images for crack detection in building pictures. Generally, the crack opening and spacing can be determined through surface displacement which is an ancient method for detection. They investigated many damage mechanisms through acoustic emission or any internal parameters [10].

Zhou et al proposed a full automated method for the detection of crack from the pavement images. To find pavement crack with higher accuracy, they used a geodesic shadow removing algorithm which is most efficient for the removal of pavement shadows during the detection process. Their pipeline work consisting of a tensor voting method for crack probability map, graph model creation for the map representation, spanning tree algorithm to detect with better accuracy. They conducted recursive tree edge pruning methods to detect the removed shadow of pavement images [11].

Talab et al examined the proposed algorithm with image processing techniques to detect the crack in the building during the concrete. They implemented a segmentation process to detect the crack images. They used Sobel filters for image segmentation. Besides, background and foreground binary subtraction filters are used for classification. They were used many filtering processes to detect the crack in the building set of images with the help of the otsu method [12].

In recent years, machine learning algorithms are used to identify the crack in the building set images. Zhang et used a single type classifier with single pixels in the image to determine the crack in an image with more details. They proposed a convolutional neural network (CNN) for image classification [13] [14]. Cha et developed a new proposed algorithm to learn fast region 
Journal of Innovative Image Processing (JIIP) (2021)

Vol.03/ No. 02

Pages: 85-99

https://www.irojournals.com/iroiip/

DOI: https://doi.org/10.36548/jiip.2021.2.002

CNN to detect a crack in the images through some edge detector algorithms such as the canny and Sobel method [15]. Also, this proposed algorithm is used to detect more types of defects in the building. They faced a problem for incompletion of the full set for crack image set. Chen et al proposed a new hybrid algorithm to fuse the CNN algorithm with the Naïve Bayes algorithm for fracture detection to improve the accuracy of detection [16].

\section{METHODOLOGIES}

\subsection{Pure Image Processing Analysis}

Image processing techniques are older, and they are continually evolving for the learning approach. We can generate binary images from grayscale images by implementing the threshold operation. After removing the noise from the input images that are impacted by noise, the binary image may be rebuilt by using morphological operations. For small objects, this eroding operation can occur after the processing stage. [17]. Different research works have used number of transformation methods to reduce the complexity in the time domain operation. They were succeeding in that by employing Fast Fourier Transform [FFT] to minimize the complexity. The Fast Fourier Transform [FFT] can be defined as,

$$
F(m, n)=\frac{1}{K L} \sum_{x=0}^{K-1} \sum_{y=0}^{L-1} f(x, y) \exp \left(-2 \pi j\left(\frac{x m}{K}+\frac{y n}{L}\right)\right)
$$

The edges of the images can be identified in the frequency domain. After applying FFT, based on the Sobel operator, the crack can be identified in the image through some edge identification. This procedure can be done with an edge detector along with convolution filters via vertical and horizontal pixel based image representation.

\subsection{Hybrid Proposed Method}

The captured image will be used as an input image and it is transformed into a gray scale image for further image analysis. The input raw images are treated with the mosaic algorithm to 
Journal of Innovative Image Processing (JIIP) (2021)

Vol.03/ No. 02

Pages: 85-99

https://www.irojournals.com/iroiip/

DOI: https://doi.org/10.36548/jiip.2021.2.002

remove overlaps and residual variation. Based on this process, an unique accuracy can be achieved. Then, the noise removal filtering processing will take place after the completion of mosaic procedure. This transformation can be derived through the following formula;

$$
f_{g s i}=\emptyset_{s e}(f)-f
$$

Where, a gray scale image is terminating from the image filtering set w.r.to structure element. The region of convergence of the image is transformed from the previous state to thresholding operation for implementing a better crack detection as shown in figure 3.

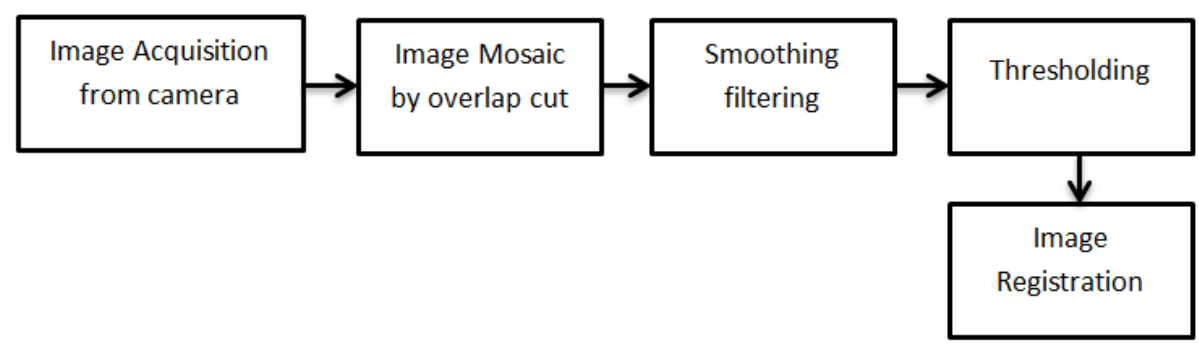

Figure 3 proposed pre-processing unit

\subsubsection{Convolutional Neural Network (CNN)}

The abstract features of the proposed algorithm's prior state in the deep learning neural network via image registration. It may be appropriate to reduce the residual variance in the images [18]. Generally, this minimization function can be performed after the squared error loss function, which is defined as follows;

$$
E s^{k}=\frac{1}{2} \sum_{i=1}^{n}\left(x_{i}^{k}-t_{i}^{k}\right)^{2}
$$

Where, $x_{i}^{k}$ “i”th target label for the sample from 1 to $n$.

CNN can perform through many convolutional and pooling layers to maximize the computation easily. The input data from the first phase of our pre-processed images can be 
Journal of Innovative Image Processing (JIIP) (2021)

Vol.03/ No. 02

Pages: 85-99

https://www.irojournals.com/iroiip/

DOI: https://doi.org/10.36548/jiip.2021.2.002

performed through many processes such as feature extraction and flattened procedure for neural computation [19]. The convolution layer function can be obtained mathematically,

$$
\begin{gathered}
c_{i}=\rho\left(x_{i}\right) \\
x_{i}=W_{i} \cdot c_{i-1}+b_{i}
\end{gathered}
$$

Where, $W_{i}$ is the convolutional layer's filters, $b_{i}$ is the bias for the every activation function in the neuron in the network. We consider the original image data size is $256 * 256$ for the convolution process to calculate the resolution of the feature map. But it can be reduced to make more information details in network and for the operation [20]. The border of the feature map is done with mirror operation in the receptive field. $256^{*} 256$ sizes can be varied in the convolutional process of the model is defined into the specified of the feature map.



Figure 4 Hybrid proposed architecture

\subsubsection{SVM Classifier}

The SVM method is fused in the CCN architecture for better classification after a trained image set. This type of accurate supervised learning will be robust for the crack identification from the dataset. We have designed for the two-class learning problems to complementary strategies for the multi-classification process. Let us consider,

$$
\left\{x_{i}, y_{i}\right\}_{i=1}^{n}
$$

ISSN: 2582-4252 (online)

Submitted: 02.05.2021

Revised: 27.05.2021

Accepted: 10.06.2021

Published: 19.06.2021 
Journal of Innovative Image Processing (JIIP) (2021)

Vol.03/ No. 02

Pages: 85-99

https://www.irojournals.com/iroiip/

DOI: https://doi.org/10.36548/jiip.2021.2.002

for the training dataset where the extracted features from the images. This regressive function can be defined as follows with mathematical,

$$
x=W \cdot c+b
$$

Here $\mathrm{c}$ is consequent layers in the proposed architecture network.

The support vector model function is used to promote the robust estimator from slight changes of the classified output. The kernel strategy is used to rectify the nonlinear task in the process of feature space with radial basis function [20]. This space is noted with higher dimensions which can be deviated to a linear function and it is expressed as,

$$
N\left(y_{i} \cdot x\right)=\exp \left(-\frac{\left\|y_{i}-x\right\|^{2}}{2 \sigma^{2}}\right)
$$

Where $\sigma$ represents a positive real number parameter.

\section{RESULTS \& DISCUSSION}

Generally, the dataset will include unique set of crack images that are obtained after the bending test of concrete structure image concrete quality of C30. The dimensions $256 * 256$ are assigned. The action is taken is based on the width of the cracks after identification. Our proposed algorithm is splitting the datasets as $80 \%$ and $20 \%$ for training and testing purposes respectively [21]. This division is testing the feasibility of our proposed algorithm. Figure 5 shows the raw input image for testing.

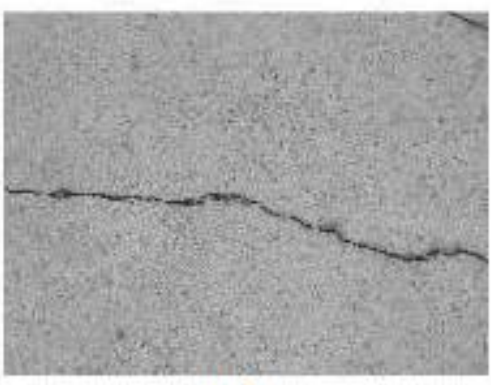

(a)

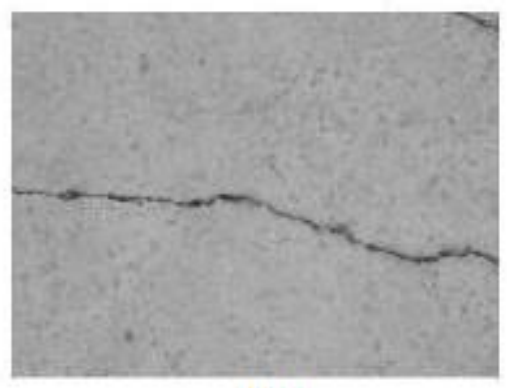

(b)

Figure 5 (a) Raw image (b) After Image mosaic for crack identification 
Journal of Innovative Image Processing (JIIP) (2021)

Vol.03/ No. 02

Pages: 85-99

https://www.irojournals.com/iroiip/

DOI: https://doi.org/10.36548/jiip.2021.2.002

From figure $5 \mathrm{~b}$, we understand the noise is reduced after the image mosaic procedure. Figure $6 \mathrm{c} \& \mathrm{~d}$ are showing the discontinuity edges can be identified through our proposed algorithm whereas figure 7 e \& f statistical and single tone classifiers are not identifying crack easily. Most of the images in the datasets are a residual variation on the distributive function. Those variation functions couldn't be detected through another existing method which is shown in figure 7. In this research article, we have measured recall, accuracy, and precision for our proposed algorithm and we obtained good results on that [22].



(c)

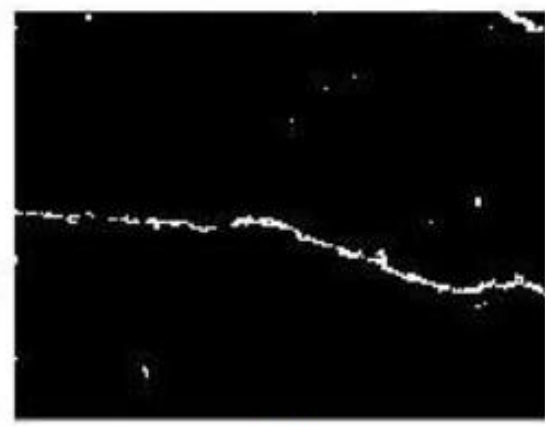

(d)

Figure 6 (c) Edge image after binary conversion (d) Proposed algorithm output

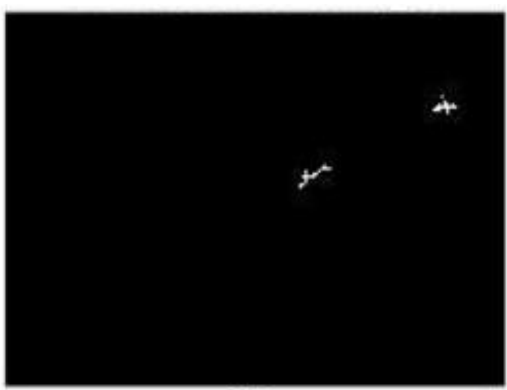

(e)

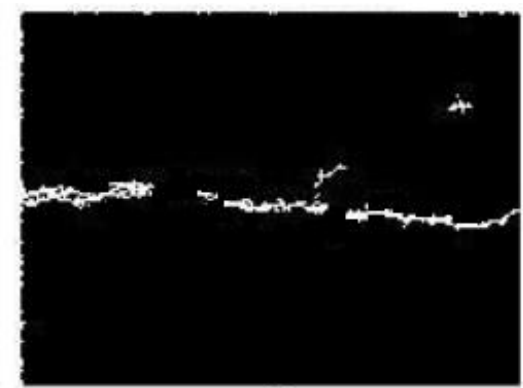

(f)

Figure 7 (e) statistical approach (f) discontinuity edges through Single type classifiers

The following formula has used to identify the proposed system feasibility.

$$
\text { Accuracy }=\frac{T P+T N}{T P+T N+F P+F N}
$$


Journal of Innovative Image Processing (JIIP) (2021)

Vol.03/ No. 02

Pages: 85-99

https://www.irojournals.com/iroiip/

DOI: https://doi.org/10.36548/jiip.2021.2.002

$$
\begin{gathered}
\text { Precision }=\frac{T P}{T P+F P} \\
\text { Recall }=\frac{T P}{T P+F N}
\end{gathered}
$$

Table 1 shows the numerical values are obtained after testing the proposed algorithm. Our proposed model has improved the precision and recall by around 10 percentage than older image processing analysis alone.

\begin{tabular}{|c|c|c|c|c|c|c|}
\hline S.No & Methods & Recall & Precision & Accuracy & $\begin{array}{c}\text { Time spent } \\
\text { on crack } \\
\text { identification }\end{array}$ & $\begin{array}{c}\text { Classification } \\
\text { error }\end{array}$ \\
\hline 1 & $\begin{array}{c}\text { Image processing } \\
\text { with FFT }\end{array}$ & $80 \%$ & $79 \%$ & $81.5 \%$ & $40 \mathrm{~S}$ & NA \\
\hline 2 & Statistical method & $86 \%$ & $77 \%$ & $80 \%$ & $49 \mathrm{~S}$ & 0.071 \\
\hline 3 & $\begin{array}{l}\text { Single classifier } \\
\qquad(\mathrm{CNN})\end{array}$ & $90 \%$ & $88 \%$ & $89 \%$ & $102 \mathrm{~S}$ & 0.053 \\
\hline 4 & $\begin{array}{c}\text { Hybrid Proposed } \\
\text { Algorithm ( CNN } \\
+ \text { SVM ) }\end{array}$ & $92 \%$ & $89.5 \%$ & $92 \%$ & $132 \mathrm{~S}$ & 0.008 \\
\hline
\end{tabular}

Table 1 comparison of proposed algorithm through obtained numerical values

Figure 8 shows the comparison for the performance of the proposed hybrid methodology for crack detection in the image. It has shown that the proposed hybrid methodology is proving that superior to other existing methods. 
Journal of Innovative Image Processing (JIIP) (2021)

Vol.03/ No. 02

Pages: 85-99

https://www.irojournals.com/iroiip/

DOI: https://doi.org/10.36548/jiip.2021.2.002

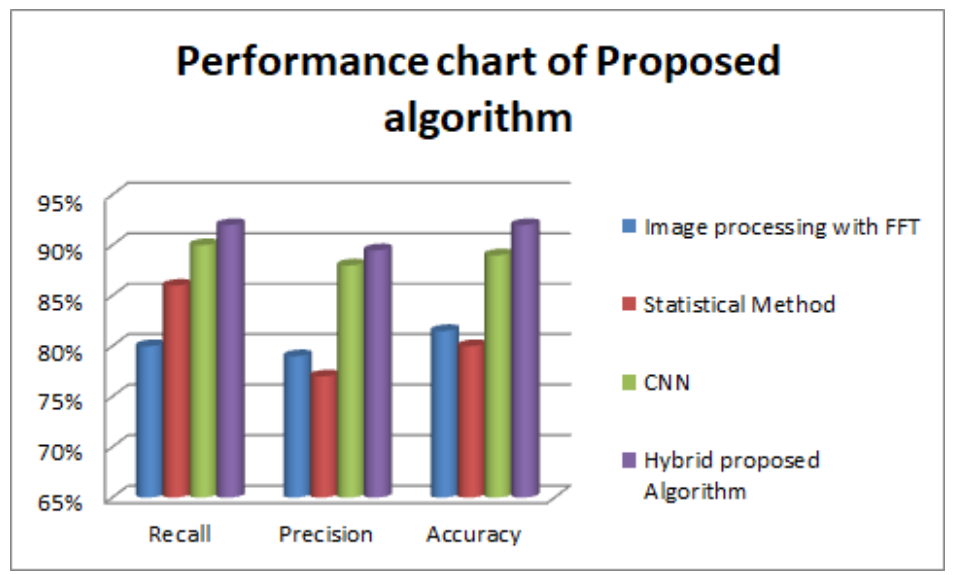

Figure 8 Comparison for performance of the proposed hybrid methodology

This comparison of the evaluation segment for image crack identification has been performed. The extra pre-processing results are relatively soft and it is reducing the false detection in the crack region. Besides, the single tone classifier learning method has been improved by pre-processing the image analysis with a fusion of another SVM method [23]. The tail of the neural network is constructed with an SVM model, which performs good classification and provides accurate detection of the crack images present in the concrete structure. Also, the pooling index procedure is connected between the pixel arranging and grouping for coarsegrained models.

\section{CONCLUSION}

Thus, our proposed hybrid identification method is examined for cracks on concrete bridge images. Besides, we presented the recent research issues on the crack detection system of concrete structures. The proposed method has leveraged better classification and fewer classification errors during the process. The recursive variation can be subsided through some noise removal in the pre-processing techniques by the proposed classification method. The width of the cracked area on the concrete structure was also calculated after it has been classified through the proposed hybrid identification method. The precision and accuracy of the test set are determined for our proposed method is around $10 \%$ higher than the existing model. The classification error is calculated and it shows least than other algorithms recently. Thus, our 
Journal of Innovative Image Processing (JIIP) (2021)

Vol.03/ No. 02

Pages: 85-99

https://www.irojournals.com/iroiip/

DOI: https://doi.org/10.36548/jiip.2021.2.002

suggested hybrid techniques outperformed other existing methods in terms of high precision, robustness, and accuracy with the least classification error in the crack identification process on concrete structures. This proposed algorithm can be extended to android based software to realize the practical engineering trends toward the $5 \mathrm{G}$ application. It is highly believed that the new integration of a superior segmentation method for image analysis will give the best accuracy for our classification in future analysis. [24]. we tested the proposed model on camera-type pictures, which provided excellent accuracy. In the future, we will enhance feature extraction approaches by conducting in-depth assessments of damage circumstances in the crack on the concrete structure. [25]. Also, various techniques of an invasive and non-invasive method for crack detection will be surveyed carefully as an extensive study.

\section{REFERENCES}

[1] Kim, Jong-Woo, Sung-Bae Kim, Jeong-Cheon Park, and Jin-Won Nam. 2015. "Development of Crack Detection System with Unmanned Aerial Vehicles and Digital Image Processing." Advances in structural engineering and mechanics (ASEM15). Inchoen, Korea: I-ASEM.

[2] Karuppusamy, P. "Building Detection using Two-Layered Novel Convolutional Neural Networks." Journal of Soft Computing Paradigm (JSCP) 3, no. 01 (2021): 29-37.

[3] Oliveira, Henrique, and Paulo L. Correia. 2013. "Automatic Road Crack Detection and Characterization." IEEE Transactions on Intelligent Transportation Systems 155-168.

[4] Shakya, Subarna, Lalitpur Nepal Pulchowk, and S. Smys. "Anomalies Detection in Fog Computing Architectures Using Deep Learning." Journal: Journal of Trends in Computer Science and Smart Technology March 2020, no. 1 (2020): 46-55.

[5] Pereira, Fábio Celestino, and Carlos Eduardo Pereira. 2015. "Embedded Image Processing Systems for Automatic Recognition of Cracks using UAVs." IFAC. PortoAlegre: ELSEVIER. 016-021.

[6] Adam, Edriss Eisa Babikir. "Survey on Medical Imaging of Electrical Impedance Tomography (EIT) by Variable Current Pattern Methods." Journal of ISMAC 3, no. 02 (2021): 82-95.

ISSN: 2582-4252 (online) 
Journal of Innovative Image Processing (JIIP) (2021)

Vol.03/ No. 02

Pages: 85-99

https://www.irojournals.com/iroiip/

DOI: https://doi.org/10.36548/jiip.2021.2.002

[7] N. D. Hoang, "Detection of surface crack in building structures using image processing technique with an improved Otsu method for image thresholding," Advances in Civil Engineering, vol. 2018, Article ID 3924120, , 2018.

[8] Haoxiang, Wang, and S. Smys. "Overview of Configuring Adaptive Activation Functions for Deep Neural Networks-A Comparative Study." Journal of Ubiquitous Computing and Communication Technologies (UCCT) 3, no. 01 (2021): 10-22.

[9] S. Santurkar, D. Tsipras, A. Ilyas, and A. Madry, "How does batch normalization help optimization?" Advances in Neural Information Processing Systems, vol. 31, pp. 2483-2493, 2018.

[10] S.Y. Alam, A. Loukili, F. Grondin, E. Rozie` re, Use of the digital image correlation and acoustic emission technique to study the effect of structural size on cracking of reinforced concrete, Eng. Fract. Mech. 143 (2015) 17-31.

[11] Qin Zou, Yu Cao, Qingquan Li, Qingzhou Mao, Song Wang, CrackTree: automatic crack detection from pavement images, Pattern Recogn. Lett. 33 (3) (2012) 227-238.

[12] A. M. A. Talab, Z. Huang, F. Xi, and L. HaiMing, "Detection crack in image using otsu method and multiple filtering in image processing techniques," Optik, vol. 127, no. 3, pp. 10301033, 2016.

[13] L. Zhang, F. Yang, and D. Zhang, "Road crack detection-using deep convolutional neural network," in Proceedings of the 2016 IEEE International Conference on Image Processing (ICIP), pp. 3708-3712, Phoenix, AZ, USA, September 2016.

[14] A. Zhang, K. C. P. Wang, B. Li et al., "Automated pixel-level pavement crack detection on 3D asphalt surfaces using a deep-learning network," Computer-Aided Civil and Infrastructure Engineering, vol. 32, no. 10, pp. 805-819, 2017.

[15] Y.-J. Cha, W. Choi, and O. B"uy"uk"ozt"urk, "Deep learning based crack damage detection using convolutional neural networks," Computer-Aided Civil and Infrastructure Engineering, vol. 32, no. 5, pp. 361-378, 2017.

[16] F. C. Chen and M. R. Jahanshahi, "NB-CNN: deep learning based crack detection using convolutional neural network and Naive Bayes data fusion," IEEE Transactions on Industrial Electronics, vol. 65, no. 5, pp. 4392-4400, 2017. 
Journal of Innovative Image Processing (JIIP) (2021)

Vol.03/ No. 02

Pages: 85-99

https://www.irojournals.com/iroiip/

DOI: https://doi.org/10.36548/jiip.2021.2.002

[17] Hariharakrishnan, Jayaram, and N. Bhalaji. "Adaptability Analysis of 6LoWPAN and RPL for Healthcare applications of Internet-of-Things." Journal of ISMAC 3, no. 02 (2021): 69-81.

[18] W. R. Crum, O. Camara, and D. L. Hill, "Generalized overlap measures for evaluation and validation in medical image analysis," IEEE Transactions on Medical Imaging, vol. 25,

no. 11, pp. 1451-1461, 2016.

[19] Adam, Edriss Eisa Babikir. "Evaluation of Fingerprint Liveness Detection by Machine Learning Approach-A Systematic View." Journal of ISMAC 3, no. 01 (2021): 16-30.

[20] L. N. Smith, "Cyclical learning rates for training neural networks," in Proceedings of the 2017 IEEE Winter Conference on Applications of Computer Vision (WACV), pp. 464-472,

Santa Rosa, CA, USA, March 2017.

[21] Adam, Edriss Eisa Babikir. "Deep Learning based NLP Techniques In Text to Speech Synthesis for Communication Recognition." Journal of Soft Computing Paradigm (JSCP) 2, no. 04 (2020): 209-215.

[22] M. A. Rahman and Y. Wang, "Optimizing intersection over- union in deep neural networks for image segmentation," in Proceedings of the International Symposium on Visual

Computing, pp. 234-244, Las Vegas, NV, USA, December 2016.

[23] Hamdan, Yasir Babiker. "Faultless Decision Making for False Information in Online: A Systematic Approach." Journal of Soft Computing Paradigm (JSCP) 2, no. 04 (2020): 226-235.

[24] Chen, Joy Iong Zong, and P. Hengjinda. "Early Prediction of Coronary Artery Disease (CAD) by Machine Learning Method-A Comparative Study." Journal of Artificial Intelligence 3, no. 01 (2021): 17-33.

[25] Ranganathan, G. "Real time anomaly detection techniques using pyspark frame work." Journal of Artificial Intelligence 2, no. 01 (2020): 20-30.

ISSN: 2582-4252 (online) 
Journal of Innovative Image Processing (JIIP) (2021)

Vol.03/ No. 02

Pages: 85-99

https://www.irojournals.com/iroiip/

DOI: https://doi.org/10.36548/jiip.2021.2.002

\section{Authors Biography}

Edriss Eisa Babikir Adam received his B.S. degree in electrical engineering from Blue Nile University, Damazein, Sudan, in 2004 and his MSc. degree in Electronics and Information Engineering from Sudan University of Science and Technology, Sudan, in 2008. Currently, he is working in Mainefhi College of Engineering and Technology, Eritrea. He has published several papers in national and international journals. His current research interests are in the areas of cooperative wireless communications, and sensor MIMO systems. He received CHINA SCHOLARSHIP COUNCIL CSC 2010 collaboration with the Sudan government.

Sathesh completed his master's degree in the year 2006 and has published several papers in national and international journals. His areas of interest include wavelets and multi-resolution transforms for image denoising. Currently, he is occupying an academic position in Eritrea after having worked in a reputed University in South India for the past 5 years. He is pursuing his research work in the area of complex wavelets for image approximations with a deep learning approach. 